\title{
Resonant Vacuum-Ultraviolet Photoelectron Spectra of Aligned Li Atoms
}

\author{
M. Meyer, ${ }^{(1)}$ B. Müller, ${ }^{(2)}$ A. Nunnemann, ${ }^{(2)}$ Th. Prescher, ${ }^{(1)}$ E. v. Raven, ${ }^{(1)}$ M. Richter, ${ }^{(1)}$ \\ M. Schmidt, ${ }^{(2)}$ B. Sonntag, ${ }^{(1)}$ and P. Zimmermann ${ }^{(2)}$ \\ ${ }^{(1)}$ II. Institut für Experimentalphysik, Universität Hamburg, D-2000 Hamburg 50, West Germany \\ ${ }^{(2)}$ Institut für Strahlungs- und Kernphysik, TU Berlin, D-1000 Berlin 33, West Germany
}

(Received 21 August 1987)

\begin{abstract}
A new method is described in which $\mathrm{Li}$ atoms are excited and aligned by pumping of the $\mathrm{Li}$ $1 s^{2} 2 s^{2} S_{1 / 2} \rightarrow 1 s^{2} 2 p^{2} P_{1 / 2,3 / 2}$ transitions with a linearly polarized dye-laser beam, and subsequently raised to core-excited $1 \operatorname{snln} l^{\prime} l^{\prime 2} S,{ }^{2} P,{ }^{2} D$ states by linearly polarized synchrotron radiation. The intensity of the electrons ejected upon the Li $1 s n \ln ^{\prime} l^{\prime 2} S,{ }^{2} D \rightarrow 1 s^{2}{ }^{1} S_{0} \epsilon l$ autoionization strongly varied with the character of the core-excited state, the intermediate ${ }^{2} P_{1 / 2}$ or ${ }^{2} P_{3 / 2}$ state, and the angle between the polarization vectors of the two photon beams.
\end{abstract}

PACS numbers: $32.80 . \mathrm{Rm}, 32.80 . \mathrm{Dz}$

Vacuum-ultraviolet (VUV) photoelectron spectroscopy has proven an ideal tool for studying the manyelectron dynamics of core-excited atoms. ${ }^{1}$ By the combining of high-power tunable lasers with synchrotron radiation, a new field has been opened. ${ }^{2-4}$ States optically inaccessible from the ground state can be reached and the ionization of excited states can be investigated. To exploit the full power of the method, we transferred the techniques developed in two-photon laser ionization ${ }^{5,6}$ to the VUV regime. The intensity and angular distribution of the outgoing photoelectrons critically depends on the character of the core-excited state, on the alignment of the intermediate state prepared by the laser, and on the relative orientation of the polarization vectors of the two

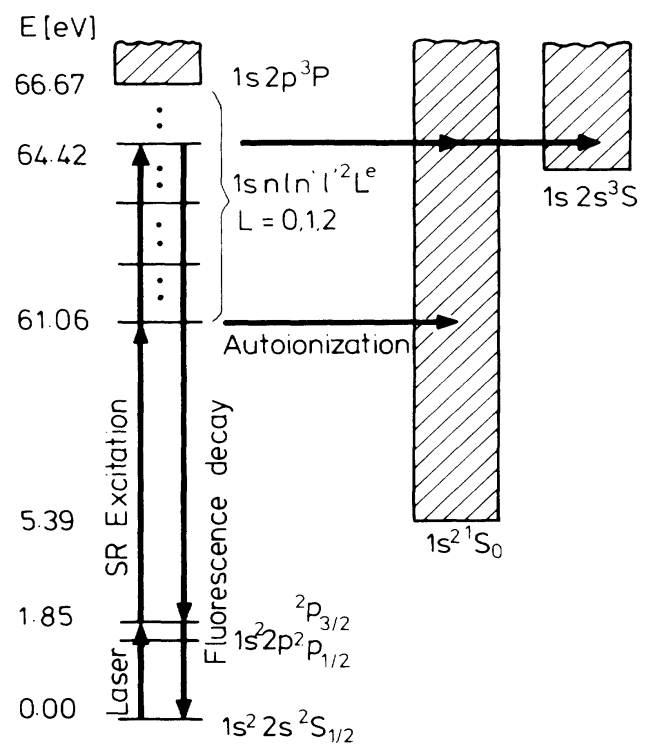

FIG. 1. Schematic energy-level diagram for the Li evenparity core-excited states reached by two-photon (laser plus synchrotron radiation) excitation. radiation fields. Therefore, two-photon ionization can be used as a very sensitive probe to study the excited states of atoms. The three-electron atom $\mathrm{Li}$ is an excellent model system to test this new method for core-excited states. The excitation and decay channels are displayed in Fig. 1. Our results are compared with the $K$ absorption spectrum of $\mathrm{Li}^{*} 1 s^{2} 2 p$ by McIlrath and Lucatorto, ${ }^{7}$ and with the assignments based on theoretical calculations. $^{8,9}$

The experimental arrangement is schematically depicted in Fig. 2. The $\mathrm{Li}$ atoms were prepared in an atomic beam emanating from a resistively heated stainless-steel oven. The oven was operated at $400^{\circ} \mathrm{C}$ to produce a density of about $10^{10} \mathrm{Li}$ atoms $/ \mathrm{cm}^{3}$ in the interaction zone. The $0.6-\mathrm{W}$ linearly polarized beam of a cw-ring-dye laser induced either the transition $2 s^{2} S_{1 / 2}$

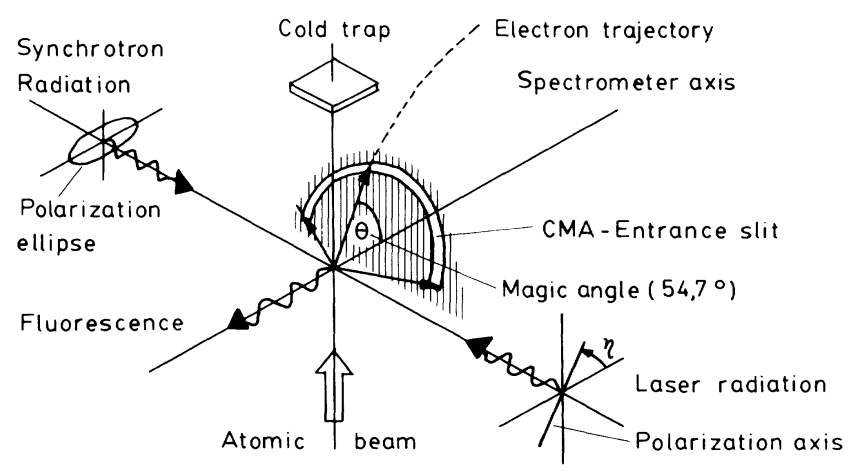

FIG. 2. Scheme of the experimental arrangement. The main axis of the polarization ellipse of the synchrotron radiation defines the $z$ axis. The entrance slit of the cylindrical-mirror analyzer, aligned along the $z$ axis, is schematically depicted. The synchrotron radiation propagates in the $y$ direction, and the laser beam in the opposite direction. Both photon beams intersect the atomic Li beam propagating in the $x$ direction. The polarization axis of the laser lies in the $x-z$ plane at an angle $\eta$ with respect to the $z$ axis. 
$\rightarrow 2 p^{2} P_{1 / 2}$ or the transition $2 s^{2} S_{1} \rightarrow 2 p^{2} P_{3 / 2}$. Doppler and saturation broadening resulted in an effective bandwidth of approximately $960 \mathrm{MHz}$. Therefore several hyperfine components were pumped simultaneously. The fractions of the excited atoms were estimated to be $3 \%$ for the ${ }^{2} P_{1 / 2}$ state and $7 \%$ for the ${ }^{2} P_{3 / 2}$ state. The resonance fluorescence emitted perpendicular to the atomic beam and the laser beams was monitored with a photomultiplier. The polarization $P$ of the resonance fluorescence was consistent with the theoretical ${ }^{10}$ value $\left[P\left({ }^{2} P_{3 / 2}\right)=0.6 ; P\left({ }^{2} P_{1 / 2}\right)=0\right]$ and with values achieved for similar systems. ${ }^{11}$ The $\mathrm{Li} \quad 1 s^{2} 2 p^{2} P_{1 / 2,3 / 2}$ $\rightarrow 1 \operatorname{sn} \ln ^{\prime} l^{\prime 2} S,{ }^{2} P,{ }^{2} D$ excitations were driven by the synchrotron radiation of the electron storage ring BESSY. The radiation was monochromatized by a toroidalgrating monochromator (bandwidth $0.2 \mathrm{eV}$, approximately $10^{11}$ photons/s). The monochromatic photon beam, linearly polarized in the horizontal plane, was focused onto the interaction zone (diameter of the focal spot $<1 \mathrm{~mm}$ ). The kinetic energy of the electrons ejected upon the decay of the core-excited states was determined by a cylindrical-mirror analyzer (angular acceptance $0.8 \%$ of $4 \pi$, energy resolution $\Delta E=0.8 \%$ of the pass energy). Only electrons emitted at angles close to the magic angle of $54.7^{\circ}$ relative to the polarization vector of the synchrotron light were accepted by the analyzer. The electron trajectory is described by the polar angles $\Theta$ and $\Phi$. The main axis of the polarization ellipse of the synchrotron radiation defines the $z$ axis. The synchrotron radiation propagates in the $y$ direction, and the atomic beam in the $x$ direction. The polarization axis of the laser lies in the $x-z$ plane at an angle $\eta$ with respect to the $z$ axis. For linearly polarized light in both photon beams, the dipole approximation for the angular distribution of the electrons is ${ }^{6}$

$$
\begin{aligned}
I(\Theta, \Phi)=\alpha_{00} P_{00}+ & \alpha_{20} P_{20}(\cos \Theta)+\alpha_{40} P_{40}(\cos \Theta)+\alpha_{21} P_{21}(\cos \Theta) \cos \Phi \\
& +\alpha_{41} P_{41}(\cos \Theta) \cos \Phi+\alpha_{22} P_{22}(\cos \Theta) \cos 2 \Phi+\alpha_{42} P_{42}(\cos \Theta) \cos 2 \Phi .
\end{aligned}
$$

The $P_{L M}(\cos \Theta)$ are the normalized associated Legendre polynomials, and the coefficients $\alpha_{L M}$ depend on $\eta$. Since our analyzer integrates over $\Phi$ from $-\pi / 2$ to $\pi / 2$, the terms containing $\cos 2 \Phi$ vanish. For $\Theta=54.7^{\circ}$, $P_{20}(\cos \Theta)$ is zero. For $\eta=0^{\circ}$ and $90^{\circ}$, only the terms $\alpha_{00} P_{00}$ and $\alpha_{40} P_{40}(\cos \Theta)$ remain. When the intermediate state is ${ }^{2} P_{1 / 2}$, the angular distribution is independent of the linear polarization direction of the laser ${ }^{12}$; therefore the term that contains $P_{40}(\cos \Theta)$ also drops out.

The radiative decay of the core-excited Li $1 \operatorname{sn}^{\prime} \ln ^{\prime} l^{\prime 2} S$, ${ }^{2} P,{ }^{2} D$ states competes with autoionization. The total VUV fluorescence emitted perpendicular to the atomic beam and to the two photon beams was monitored by an open-faced solar-blind photomultiplier. A negative voltage applied to a fine mesh, mounted in front of the multiplier, prevented electrons from reaching the CsI cathode. Positive ions did not give rise to a noticeable background. Further details of the experimental setup are given by Nunnemann et al. ${ }^{4}$

For photon energies between 58.5 and $64.5 \mathrm{eV}$, the intensity of the electrons emitted upon the $\mathrm{Li} 1 \operatorname{snln}^{\prime} l^{\prime}$ $\rightarrow 1 s^{2}{ }^{1} S \epsilon l$ decay is shown in Fig. 3. The Li $1 s n l n^{\prime} l '$ states were excited via ${ }^{2} P_{1 / 2}$ [that is, $\mathrm{Li}$ $1 s^{2} 2 s^{2} S_{1 / 2} \rightarrow 1 s^{2} 2 p^{2} P_{1 / 2} \rightarrow 1 s n \ln ^{\prime} l^{\prime}$ (see Fig. 1)]. The experimental data points are fitted by a superposition of Gaussian profiles having $0.2 \mathrm{eV}$ half-width. The experimental data have been normalized to the incoming photon flux and corrected for the energy dependence of the bandpass of the electron energy analyzer. In agreement with theory, the spectrum did not change when the polarization vector of the laser was turned from the perpendicular to the parallel direction with respect to the polarization vector of the synchrotron radiation. Therefore, the spectrum represents the partial autoionization cross section. The stick diagram gives the relative strengths of the $1 s^{2} 2 p^{2} P \rightarrow 1 s n \ln ^{\prime} l^{\prime}$ absorption lines read off the absorption spectrum reported by McIlrath and Lucatorto. ${ }^{7}$ They have been normalized to our data by the matching of the amplitudes of line 5. Reading line strengths from photographically recorded spectra entails considerable error. Therefore, only qualitative agreement is expected. The energies and the assignment of the lines given by McIlrath and Lucatorto, ${ }^{7}$ Berry, ${ }^{9}$ and Bunge ${ }^{8}$ are summarized in Table I. The even-parity Li $1 \operatorname{snln}^{\prime} l^{\prime 2} P$ states

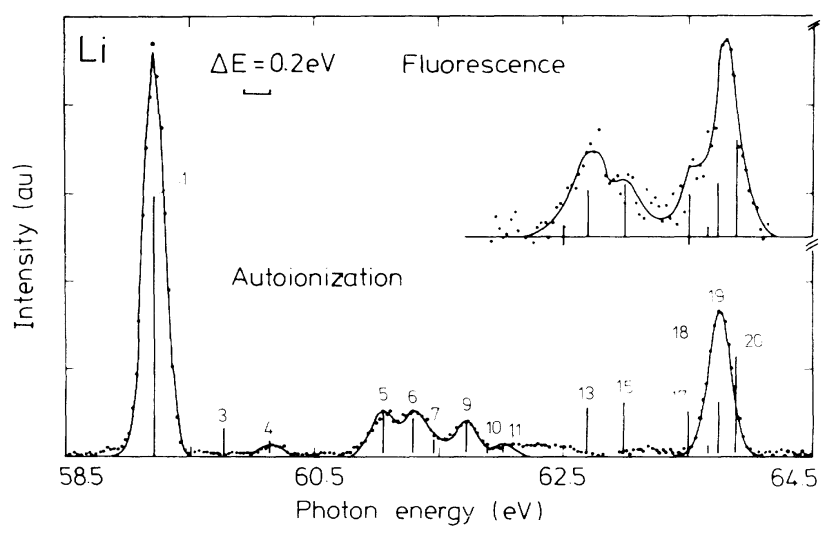

FIG. 3. Intensity of the electrons emitted upon the $\mathrm{Li}$ $1 s^{2} 2 s^{2} S_{1 / 2} \rightarrow 1 s^{2} 2 p^{2} P_{1 / 2} \rightarrow 1 s n l n^{\prime} l^{\prime} \rightarrow 1 s^{2} S_{0}+\epsilon l$ excitation and autoionization sequence. The stick diagram gives the relative strengths of the $1 s^{2} 2 p^{2} P \rightarrow 1 s n \ln ^{\prime} l^{\prime}$ absorption lines detected by McIlrath and Lucatorto (Ref. 7). Inset: Total VUV fluorescence yield for photon energies between 61.5 and $64.5 \mathrm{eV}$. 
TABLE I. Line numbers (see Fig. 3 and 4), energies, and assignment for the transitions $\mathrm{Li}$ $1 s^{2} 2 p^{2} P \longrightarrow 1$ snln' $l^{\prime}$ from (a) Mcllrath and Lucatorto (Ref. 7); (b) this work; (c) Bunge (Ref. 8); and (d) Berry (Ref. 9).

\begin{tabular}{|c|c|c|c|c|}
\hline \multirow{2}{*}{$\begin{array}{c}\text { Line } \\
\text { number }\end{array}$} & \multicolumn{2}{|c|}{$\hbar \omega(\mathrm{eV})$} & \multicolumn{2}{|r|}{ Assignment } \\
\hline & (a) & (b) & (a) & (c) and (d) \\
\hline 1 & 59.21 & $59.21(1)$ & $1 s\left(2 p^{2}\right)^{2} D$ & \\
\hline 2 & 59.69 & & & \\
\hline 3 & 59.77 & & $1 s\left(2 p^{2}\right)^{2} P$ & $1 s 2 p^{22} P(\mathrm{c})$ \\
\hline 4 & 60.14 & $60.16(2)$ & $1 s 2 s\left({ }^{3} S\right) 3 s^{2} S$ & \\
\hline 5 & 61.05 & $61.06(2)$ & $1 s 2 s\left({ }^{3} S\right) 3 d^{2} D$ & \\
\hline 6 & 61.29 & $61.29(2)$ & $1 s 2 s\left({ }^{1} S\right) 3 s^{2} S$ & \\
\hline 7 & 61.46 & $61.46(2)$ & $1 s 2 s\left({ }^{3} S\right) 4 s^{2} S$ & \\
\hline 8 & 61.61 & & $1 s\left(2 p^{2}\right)^{2} S$ & \\
\hline 9 & 61.72 & $61.73(2)$ & $1 s 2 s\left({ }^{3} S\right) 4 d^{2} D$ & $(1 s 2 s 5 p){ }^{4} P(\mathrm{~d})$ \\
\hline 10 & 61.89 & & $1 s 2 s\left({ }^{3} S\right) 5 s^{2} S$ & \\
\hline 11 & 62.02 & $62.02(2)$ & $1 s 2 s\left({ }^{3} S\right) 5 d^{2} D$ & \\
\hline 12 & 62.09 & & & \\
\hline 13 & 62.70 & & $1 s 2 s\left({ }^{1} S\right) 3 d^{2} D$ & \\
\hline 14 & 62.74 & & $1 s 2 p\left({ }^{3} P\right) 3 p^{2} P$ & $(1 s 2 p)^{3} P 3 p^{2} P(\mathrm{c})$ \\
\hline 15 & 62.99 & & $1 s 2 p\left({ }^{3} P\right) 3 p^{2} D$ & $(1 s 2 p 3 p){ }^{4} P(\mathrm{~d})$ \\
\hline 16 & 63.16 & & & \\
\hline 17 & 63.52 & & $1 s 2 s\left({ }^{1} S\right) 4 d^{2} D$ & \\
\hline 18 & 63.67 & & $1 s 2 p\left({ }^{1} P\right) 3 p^{2} P$ & {$\left[1 s 2 p\left({ }^{1} P\right) 3 p+1 s 2 p\left({ }^{3} P\right) 4 p\right]{ }^{2} P(\mathrm{c})$} \\
\hline 19 & 63.75 & $63.75(2)$ & $1 s 2 p\left({ }^{1} P\right) 3 p^{2} D$ & \\
\hline 20 & 63.89 & & $1 s 2 s\left({ }^{1} S\right) 5 d^{2} D$ & {$\left[1 s 2 p\left({ }^{3} P\right) 4 p-1 s 2 p\left({ }^{1} P\right) 3 p\right]{ }^{2} P(\mathrm{c})$} \\
\hline
\end{tabular}

are metastable against autoionization. This results from a combination of parity and angular-momentum selection rules. It explains why there is no line 3 in the autoionization spectrum. The intensity of the VUV fluorescence radiation as a function of the energy of the exciting photon is shown in the inset to Fig. 3. The lines 13, 15 , and 17 , which are missing in the electron spectrum, clearly show up in the fluorescence spectrum. This demonstrates that the core-excited states decay via photon emission. These states also can autoionize into the $1 s 2{ }^{3} S \epsilon l$ continuum (see Fig. 1). The low-energy electrons emitted upon the $1 s n l n^{\prime} l^{\prime} \rightarrow 1 s 2 s^{3} S \epsilon l$ autoionization could not be detected in our experiment. At the energy of lines 19 and 20 , there is also a strong fluorescence signal. It is obvious that radiative decay of the core-excited state responsible for absorption line 20 contributes to the fluorescence. However, the center of the fluorescence line is very close to the position of line 19. This indicates either that the core-excited state of line 19 decays via photon emission and autoionization into the $1 s^{2}{ }^{1} S \epsilon l$ continuum, or that another state, metastable against autoionization, lies very close by.

The comparison of the electron, fluorescence, and absorption spectra allowed us to single out states which are metastable against $1 s n l n^{\prime} l^{\prime} \rightarrow 1 s^{2}$ 'S $\epsilon l$ autoionization. In order to discriminate between the ${ }^{2} S$ and ${ }^{2} D$ states, we made use of the alignment of the $1 s^{2} 2 p^{2} P_{3 / 2}$ intermediate state. Figure 4 shows three $1 s n l n^{\prime} l^{\prime} \rightarrow 1 s^{2}{ }^{1} S \epsilon l$ autoionization electron spectra of the lines 5-9 measured with a reduced monochromator bandpass of $0.12 \mathrm{eV}$. The uppermost spectrum gives the absorption lines ${ }^{7}$ convoluted by Gaussians having $0.12 \mathrm{eV}$ half-width. The partial-autoionization cross section for the excitation via ${ }^{2} P_{1 / 2}$ is represented by the second spectrum. This spectrum closely resembles the absorption spectrum. For the two remaining spectra, the autoionizing core-excited states have been reached via the $1 s^{2} 2 p^{2} P_{3 / 2}$ state. The polarization vectors of the laser light and the synchrotron radiation were oriented parallel to one another in the case of spectrum 3, and perpendicular to one other in case of spectrum 4. The solid line represents a superposition of Gaussians (FWHM $=0.12 \mathrm{eV}$ ) fitted to the experimental data. Within the accuracy of our measurements, the amplitude of line 5 is the same in spectra 2, 3, and 4. In contrast, the amplitudes of the lines 6, 7, and 9 vary dramatically upon a change of the intermediate state and the relative polarization of the two light beams. For maximum alignment of the ${ }^{2} P_{3 / 2}$ intermediate state, the intensity of $1 s^{2} 2 p^{2} P_{3 / 2} \rightarrow 1 s n l n^{\prime} l^{\prime 2} D$ and $1 s^{2} 2 p$ ${ }^{2} P_{3 / 2} \rightarrow 1 s n l n^{\prime} l^{\prime 2} S$ transitions are expected to increase by a factor of 1.1 and 2, respectively, compared with the intensity of the transitions to the same final state from the $1 s^{2} 2 p^{2} P_{1 / 2}$ intermediate state. ${ }^{13}$ This is corroborated by the intensity changes of lines $5-7$, whereas line 9 , which is assigned to a transition to a ${ }^{2} D$ state, ${ }^{7}$ behaves like a transition to a ${ }^{2} S$ state. Orienting the laser polarization vector perpendicular to the polarization vector of the synchrotron radiation results in a different popula- 


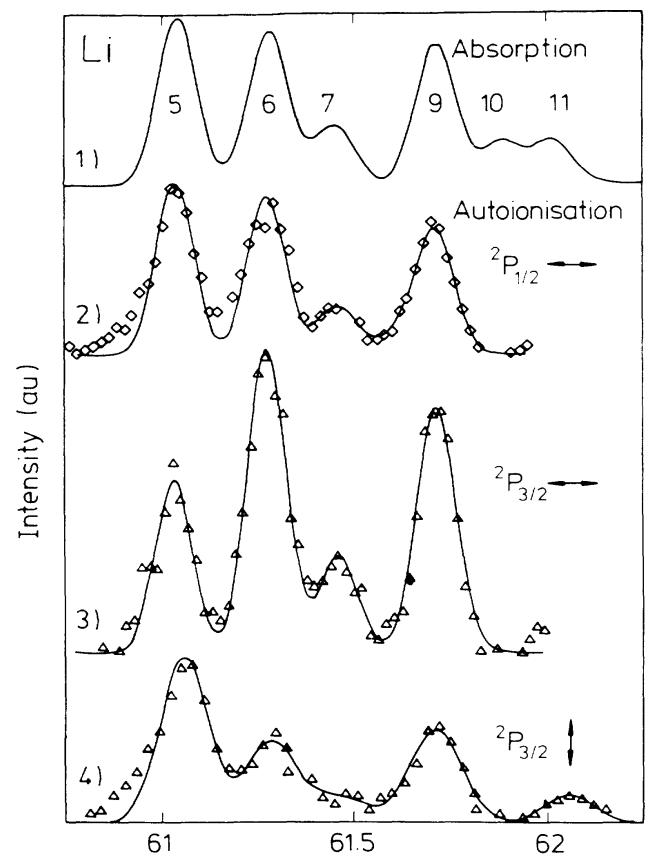

FIG. 4. $\mathrm{Li}^{*} 1 s^{2} 2 p^{2} P \rightarrow 1 s n l n^{\prime} l^{\prime}$ absorption (Ref. 7) (uppermost curve) and partial-autoionization cross section for excitation via the ${ }^{2} P_{1 / 2}$ (spectrum 2 ) and ${ }^{2} P_{3 / 2}$ (spectra 3 and 4 ) intermediate state. The polarization vector of the laser was oriented parallel to the polarization vector of the synchrotron radiation for spectra 2 and 3 and perpendicular for spectrum 4 .

tion of the $m$ sublevels of the intermediate ${ }^{2} P_{3 / 2}$ state seen by the synchrotron radiation. This population can be obtained by a transformation of the coordinate system. Consistent with the experimental result we get a very small change for the ${ }^{2} P_{3 / 2} \rightarrow{ }^{2} D_{3 / 2,5 / 2}$ transitions but a reduction by a factor of 4 for the ${ }^{2} P_{3 / 2} \rightarrow{ }^{2} S_{1 / 2}$ transitions. Line 9 clearly is due to a ${ }^{2} P \rightarrow{ }^{2} S$ transition.
VUV photoelectron spectroscopy of laser-aligned free atoms is a new method for the study of core-excited states. The wealth of detailed information accessible is showcased by our results.

The authors thank the BESSY staff for their continued assistance. The financial support of the Bundesministerium für Forschung und Technologie is gratefully appreciated.

${ }^{1}$ M. Meyer, Th. Prescher, E. v. Raven, M. Richter, M. Schmidt, B. Sonntag, and H.-E. Wetzel, Z. Phys. D 2, 347 (1986).

2J. M. Bizau, F. Wuilleumier, P. Dhez, D. L. Ederer, J. L. Picqué, J. L. Gouet, and P. Koch, in Laser Techniques for Extreme Ultraviolet Spectroscopy, edited by T. J. McIlrath and R. J. Freeman, AIP Conference Proceedings No. 90 (American Institute of Physics, New York, 1982), p. 331.

${ }^{3}$ J. M. Bizau, F. Wuilleumier, D. L. Ederer, J. C. Keller, J. L. Le Gouet, J. L. Picqué, B. Carré, and P. M. Koch, Phys. Rev. Lett. 55, 1281 (1985).

${ }^{4}$ A. Nunnemann, Th. Prescher, M. Richter, M. Schmidt, B. Sonntag, H.-E. Wetzel, and P. Zimmermann, J. Phys. B 18, L337 (1985).

${ }^{5}$ A. Siegel, J. Ganz, and H. Hotop, J. Phys. B 16, 2945 (1983).

${ }^{6}$ J. C. Hansen, I. A. Duncanson, R. Chien, and R. S. Berry, Phys. Rev. A 21, 222 (1980).

${ }^{7}$ T. J. McIlrath and T. B. Lucatorto, Phys. Rev. Lett. 38, 1390 (1977).

${ }^{8}$ C. F. Bunge, Phys. Rev. A 19, 936 (1979).

${ }^{9}$ H. G. Berry, Phys. Scr. 12, 5 (1975).

${ }^{10} \mathrm{Ch}$. H. Greene and R. N. Zare, Annu. Rev. Phys. Chem. 33, 119 (1982).

${ }^{11}$ A. Fischer and I. V. Hertel, Z. Phys. A 304, 103 (1982).

12J. A. Duncanson, M. P. Strand, A. Lindgård, and R. S. Berry, Phys. Rev. Lett. 37, 987 (1976).

${ }^{13}$ M. M. Lambropoulos and R. S. Berry, Phys. Rev. A 8, 855 (1973). 